\title{
De novo donor-specific antibody without rejection does not always predict worse outcome in kidney transplantation
}

\author{
Hyo Kee Kim, Sangil Min, Chris Tae Young Chung, Hyunmin Ko, Kwang Woo Choi, Ahram Han, \\ Sanghyun Ahn, Jongwon Ha
}

Division Transplantation, Department of Surgery, Seoul National University Hospital, Seoul, Korea

Background: De novo donor-specific antibodies (dnDSA) have been reported as a risk factor for graft injury and failure in kidney transplantation. However, there are only a few studies on how to manage the patients who have dnDSA without any rejection episodes. We compared the long-term results of patients with dnDSA with respect to the occurrence of biopsy proven rejection.

Methods: From January 2010 to December 2017, total of 1,172 patients received kidney transplantation in Seoul National University Hospital. Among the cohort, we included patients who had both dnDSA and kidney biopsy and classified the patients into groups according to the state of rejection.

Results: A total of 53 patients had dnDSA with biopsy. Among them, 28 patients had rejection (Rejection group), 13 patients had borderline rejection and 12 patients had no pathological abnormality (No rejection group). One-year and 3-year eGFR (estimated glomerular filtration rate) from detected dnDSA were not different between Rejection group and No rejection group (Rejection vs. No rejection: 1-year eGFR, $49.00 \pm 19.56 \mathrm{~mL} / \mathrm{min} / 1.73 \mathrm{~m}^{2}$ vs. $42.17 \pm 23.37 \mathrm{~mL} / \mathrm{min} / 1.73 \mathrm{~m}^{2}, \mathrm{P}=0.403 ; 3$-year eGFR, $46.62 \pm 29.86$ $\mathrm{mL} / \mathrm{min} / 1.73 \mathrm{~m}^{2}$ vs. $\left.49.28 \pm 20.44 \mathrm{~mL} / \mathrm{min} / 1.73 \mathrm{~m}^{2}, \mathrm{P}=0.861\right)$. However, there was a difference in graft survival between the Rejection group and the No rejection group (3-year survival: $80 \%$ vs. $100 \%, P=0.048$ ). In the multivariate analysis, recipients age (hazard ratio [HR], 0.927; 95 confidence interval [Cl], 0.882-0.974; $\mathrm{P}=0.003)$ and ATMR (HR, 10.631; $95 \% \mathrm{Cl}, 1.931-58.539 ; \mathrm{P}=0.007)$ were significant risk factors for graft failure, but there was no association between DSA (class I/II, sum of MFI, persistence of DSA, days until DSA detection) and graft failure.

Conclusions: The prognosis of patients with dnDSA but no rejection episodes within 6 months was better than those with rejections. These results suggest that dnDSA alone cannot always predict worse long-term outcome but further larger prospective studies are needed to support this conclusion. 